\title{
Study About the Despeckling Methods for Retinal Optical Coherence Tomography Images
}

\author{
Pradeep Gupta $^{1}$, Shyam Lal ${ }^{* 2}$ and Farooq Husain ${ }^{1}$ \\ ${ }^{1}$ Department of Electronics and Communication Engineering, Moradabad Institute of Technology, India \\ ${ }^{2}$ Department of Electronics and Communication Engineering, National Institute of Technology Karnataka, India
}

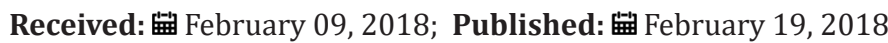

*Corresponding author: Shyam Lal, Department of Electronics and Communication Engineering, National Institute of Technology Karnataka, Surathkal, Mangaluru-575025, Karnataka, India

\begin{abstract}
This paper presents study about the different despeckling methods used for quality enhancement of retinal optical coherence tomography (OCT) images. Speckle noise is an inherent property of an OCT images which affects the visual quality of the images, hence difficult to diagnosis the patients. Therefore speckle noise reduction from the OCT images is an important prerequisite, whenever OCT imaging is used for diagnosis. The speckle noise intensity depends on the various imaging system parameters of its systems and the different structure representations used for the image tissues. A despeckling technique is to be designed in such a way that it should be able to reduce the speckle noise from the OCT images while preserving the tissues and fine details of the images.
\end{abstract}

Keywords: Despeckling; Optical coherence tomography; Speckle noise

\section{Introduction}

Optical Coherence Tomography (OCT) is a most powerful biomedical imaging technique used to extract the required information from an object. The working approach of this method is almost similar to ultrasound imaging except the medium used in obtaining its. OCT imaging uses light beams instead of sound [1]. OCT has proved its significant importance in the field of ophthalmology, especially in the detection of diseases related to retina and glaucoma [2-4]. Currently OCT is a best suitable method for imaging the internal structure of biological systems and also an essential part of a procedure to obtain high-resolution images of the retina [5-7]. It is also observe that retinal layer thickness of the OCT images improve the clinical finding in the field of ophthalmology. Retinal layer segmentation improves the clinical finding in the case of glaucoma progression and macular degeneration. Therefore, the pre-processing of ophthalmic OCT images is a most importance step to improve the clinical diagnosis. Ophthalmic OCT imaging technique is based on detection of coherent waves; therefore these images are accompanied with a significant amount of speckle noise, which degrade the image quality and limit the contrast to noise and signal to noise ratio of the image.
To reduce the effect of speckle noise and to preserve the fine image features. A good speckle noise reduction algorithm is required that reduce the effect of speckle noise while preserving fine details of image. A lot of efforts have been made to overcome the effect of speckle noise. All methods proposed for speckle noise reduction from the OCT images are mainly divided into two categories namely transform domain techniques and spatial domain techniques. Organization of manuscript is as follows: section-2: describes brief about speckle noise; section-3: presents example of speckle noise reduction from the OCT image; section-4: discusses about different despeckling methods for OCT images and its summary is also presented in tabular form; section-5: presents summary and conclusion of the manuscript.

\section{Speckle Noise}

When a signal or an image is acquired by narrowband detection systems like SAR, ultrasound and OCT, then a pattern known as speckle effect the quality of these objects. The main reasons that can affect the speckle are optical properties of the system, status of the subject, size and coherence nature of source, multiple scattering, 
phase difference of the beam and aperture of the detector $[8,9]$. Further we can classify the speckle present in OCT images into two categories first one signal- carrying speckle and the second one is signal- degrading speckle .The mathematical distribution of the speckle can be model with a Rayleigh distribution. In this modelling, speckle noise assumed as a multiplicative behaviour, in contrast to additive nature of the noise. The mathematical model of
Speckle noise can be represented as:

$$
Y(m, n)=X(m, n) S(m, n)
$$

Where $\mathrm{Y}, \mathrm{X}$ and $\mathrm{S}$ represent the noisy image data, original image data and speckle noise respectively. Normally, the multiplicative nature of speckle noise is converting into additive nature by logarithmic transformation of equation (1).

\section{Example of Noise Reduction in OCT image}

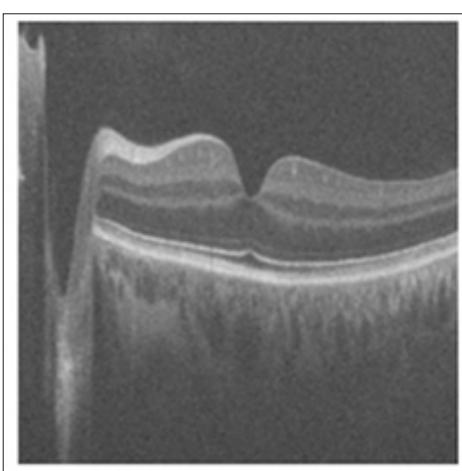

(a) Noisy Image

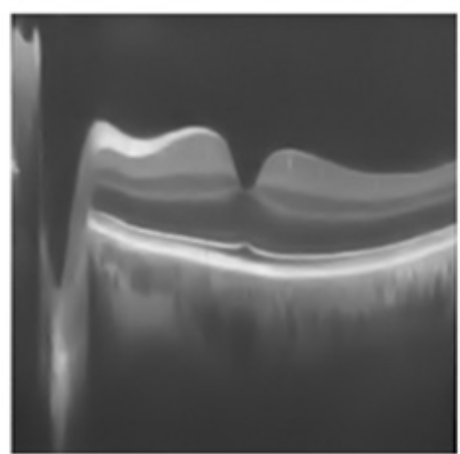

(b) Output of DTCWT[16]

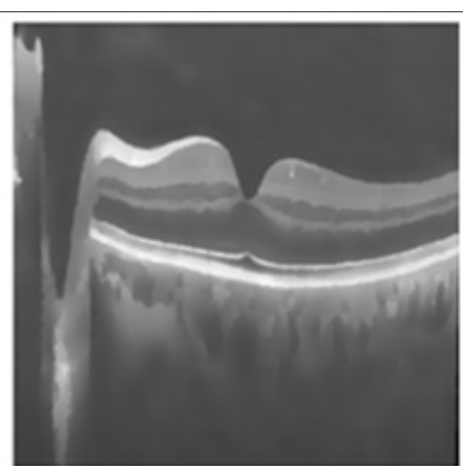

(c) Output of TGV [16]

Figure 1: Speckle Noise Reduction from the Noisy Image.

A noise reduction technique is required to improve the visual quality of the OCT image. All noise reduction techniques can be implemented into two ways, first one implemented during the acquisition time and second one implemented after acquisition of images. Normally, the noise reduction technique during the acquisition time is not good solution because these techniques require repetition of the same data which increase the acquisition time. Therefore, the use of post-processing techniques is more favourable solution to reduce the speckle noise from the OCT images. The example of speckle noise reduction from the noisy image is shown in the Figure 1. In this Figure 1(a) is the noisy image, 1(b) is the output image of DTCWT method [9] and 1(c) is the output image of second order total generalized variation (TGV) [9] method. From the Figure 1, it is clear that the effect of speckle noise reduction is very clear.

\section{Despeckling methods}

In the literature, a number of researchers proposed different post-processing methods to overcome the effect of for speckle noise from the OCT images. Few classic despeckling techniques that are used for SAR images, successfully used for OCT images also which are Lee [10], kuan [11] and frost filters [12]. An image restoration method is introduced that overcome the limitations of medical tomographic imaging system, by recovering the noiseless high frequency information corrupted by imaging system [13]. Especially for the multi-dimensional image data a better technique, based on compressive sensing principles is developed. This technique has capability to reduce the speckle noise while interpolate the missing data. This technique termed sparsity based simultaneous denoising and interpolation (SBSDI) [14]. A more appropriate approach based on dual-tree complex wavelet transform (DT-CWT) has been developed to overcome the effect of speckle noise from the OCT images. In this process adaptive-weighted bilateral filter (AWBF) is use to further enhance the OCT imaging by use of smoothing process [15].

For better image representation a new multiscale geometric analysis tool knows as wave atom transform was also proposed. The wave atom transform give better visual results in comparison to other transform such as wavelet and curvelet transform [16]. Image decomposition may also help in image denoising if the speckle noise in the OCT image is consider as texture or oscillatory patterns. A second order total generalized variation (TGV) decomposition model is helpful to remove the texture from the OCT image [16]. TGV is also removing the staircase side effect from the resulted images. Adaptive bilateral filter is also help to enhance the multi-frame OCT data [17]. The processed frames are averaged to convert into denoised output OCT image [17]. Variational image decomposition is also used to reduce the speckle noise from the ophthalmic OCT images [18].

The method converts the original image into cartoon part, texture part and speckle noise part to reduce the speckle noise from the image [18]. This method has the capability to suppress the speckle noise, while preserve image features such as edges and texture [18]. It may be useful for edge detection, segmentation and thickness calculation [18]. Independent Component analysis (ICA) techniques are also used in noise reduction of retinal OCT images [19]. The ICA technique can be beneficial when fewer number of B-scans images are available [19]. In, Raheleh kafieh et al. [20] proposed method for speckle denoising of OCT images based on 
dictionary learning approach with dual tree complex wavelet transform. In Zahra Amini [21], and Hossein Rabbani proposed method for speckle denoising of OCT images based Nonlinear transform based approach. To design the structural dictionaries, the information related to retinal layer is extracted automatically in the SSR method [22].

This method also uses the patch similarity index to enhance the performance [22]. Recently, optimization approach based on MAP

Table 1: Summary of different despeckling methods for OCT Images. estimate is proposed [23]. It use a suitable noise reduction approach along with Huber variant of TVR that overcome the effect of speckle noise, while preserve the edges as well [23]. To reduce the speckle noise, selection of a digital filter is a very difficult task. To overcome this limitation a framework based on learning approach known as learnable despeckling framework (LDF) has been proposed [24]. This approach only uses a single quality metric for selecting the appropriate filter for the task [25]. The different despeckling techniques are also studied and summarize into a Table 1.

\begin{tabular}{|c|c|c|c|c|}
\hline Referene & Approach & Method & Advantages & Limitations \\
\hline N. Anatrasirichai et al. [14] & $\begin{array}{l}\text { a. Use intensity adjustment } \\
\text { with wavelet. } \\
\text { b. Filter weight are adaptive } \\
\text { using local entropy of the } \\
\text { image. }\end{array}$ & $\begin{array}{l}\text { a Use Dual tree complex } \\
\text { wavelet transform (DT- } \\
\text { CWT) with Adaptive - } \\
\text { weighted bilateral filter } \\
\text { b. Cauchy Distribution is } \\
\text { used for removal of the } \\
\text { spatially correlated speckle. } \\
\text { c. Shape of spatial filter } \\
\text { governs by local entropy. }\end{array}$ & $\begin{array}{l}\text { a. Improved directional } \\
\text { selectivity by using } \\
\text { multiscale-based } \\
\text { segmentation } \\
\text { b. Improvement in } \\
\text { glaucoma detection. }\end{array}$ & $\begin{array}{l}\text { Use only for Glaucoma } \\
\text { detection }\end{array}$ \\
\hline Yongzhao Du et al. [15] & $\begin{array}{l}\text { A Shrinkage Filter Based on } \\
\text { Wave atom transforms. }\end{array}$ & $\begin{array}{l}\text { Wave atoms approach with } \\
\text { cycle spinning technology. }\end{array}$ & $\begin{array}{l}\text { a. Preserve the all image } \\
\text { features. } \\
\text { b.Only an adjustable } \\
\text { parameter controls the } \\
\text { noise intensity. }\end{array}$ & High complexity. \\
\hline Raheleh kafieh et al. [20] & Complex based K-SVD & $\begin{array}{c}\text { Based on dictionary } \\
\text { learning approach with } \\
\text { dual tree complex wavelet } \\
\text { transform. }\end{array}$ & $\begin{array}{c}\text { Adaptability in dictionary } \\
\text { learning }\end{array}$ & Time complexity is very high. \\
\hline Jinming Duan et al. [16] & $\begin{array}{c}\text { Total Generalized Variation } \\
\text { (TGV) based decomposition } \\
\text { model. }\end{array}$ & $\begin{array}{l}\text { Used Bregman iterative } \\
\text { parameter of Bregman } \\
\text { algorithm along with Fast } \\
\text { Fourier Transform. }\end{array}$ & $\begin{array}{l}\text { Remove staircase side effect } \\
\text { from the resulted image. }\end{array}$ & $\begin{array}{l}\text { Computational efficiency } \\
\text { increase }\end{array}$ \\
\hline P.V. Sudeep et al. [17] & $\begin{array}{l}\text { Approach based on bilateral } \\
\text { filtering. }\end{array}$ & $\begin{array}{l}\text { a. Use Gamma noise model. } \\
\text { b.Conventional bilateral } \\
\text { filter enhance the multi- } \\
\text { frame OCT data. }\end{array}$ & $\begin{array}{l}\text { a. Considerable speckle } \\
\text { noise reduction. } \\
\text { b. Better preservation }\end{array}$ & $\begin{array}{l}\text { Assumption of better } \\
\text { performance of bilateral } \\
\text { filter. }\end{array}$ \\
\hline Hongwei Ren et al. [18] & $\begin{array}{c}\text { Variational image } \\
\text { decomposition approach }\end{array}$ & $\begin{array}{l}\text { a. Cartoon part, texture part } \\
\text { and speckle noise part are } \\
\text { extracted. } \\
\text { b. Adaptive Hilbert space } \\
\text { describes the texture part. } \\
\text { c. Curvelet space describes } \\
\text { the speckle noise part. }\end{array}$ & $\begin{array}{l}\text { Useful tool for future } \\
\text { in edge detection, } \\
\text { segmentation and layer } \\
\text { thickness evaluation. }\end{array}$ & $\begin{array}{l}\text { Artificial texture increase } \\
\text { hence difficult the image } \\
\text { analysis. }\end{array}$ \\
\hline
\end{tabular}




\begin{tabular}{|c|c|c|c|c|}
\hline $\begin{array}{c}\text { Ahmadreza Baghaie et al. } \\
\text { [19] }\end{array}$ & Used ICA technique & $\begin{array}{l}\text { ICA techniques, RUNICA, } \\
\text { JADE, Fast ICA and SOBI are } \\
\text { studied }\end{array}$ & $\begin{array}{l}\text { a. JADE has a quadratic } \\
\text { behaviour. } \\
\text { b. SOBI is the best among } \\
\text { the ICA techniques }\end{array}$ & $\begin{array}{l}\text { Computational efficiency } \\
\text { must be considered. }\end{array}$ \\
\hline $\begin{array}{c}\text { Zahra Amini, Hossein } \\
\text { Rabbani } \\
{[21]}\end{array}$ & $\begin{array}{l}\text { Nonlinear transform based } \\
\text { approach. }\end{array}$ & $\begin{array}{l}\text { a. Gaussianization, process } \\
\text { converts the probability } \\
\text { distribution function into a } \\
\text { Gaussian distribution. } \\
\text { b. Normal-Laplace mixture } \\
\text { distribution decides the } \\
\text { histogram. }\end{array}$ & $\begin{array}{c}\text { a.Gaussian assumption } \\
\text { gives more accurate results. } \\
\text { b.Improve segmentation } \\
\text { accuracy of intra retinal } \\
\text { layer }\end{array}$ & $\begin{array}{l}\text { a. Presence of } \\
\text { b.Cysts, edemas, drusen,or } \\
\text { other pathologies create a } \\
\text { problem. }\end{array}$ \\
\hline Leyuan Fang et al. [22] & $\begin{array}{l}\text { Sparse reconstruction } \\
\text { method }\end{array}$ & $\begin{array}{l}\text { a. Design the structural } \\
\text { dictionaries. } \\
\text { b. Find the similar patch }\end{array}$ & $\begin{array}{c}\text { a. Used for layer } \\
\text { segmentation. } \\
\text { b. Better representation }\end{array}$ & $\begin{array}{c}\text { Fixed size of rectangular } \\
\text { patch. }\end{array}$ \\
\hline Muxingzi Li et al. [23] & $\begin{array}{l}\text { Numerical optimization } \\
\text { framework }\end{array}$ & $\begin{array}{l}\text { a. It is use maximum-a- } \\
\text { posterior estimate. } \\
\text { b. Edges are preserve by } \\
\text { using Huber variant }\end{array}$ & $\begin{array}{l}\text { a.Less costly } \\
\text { b.Better performance than } \\
\text { K-SVD }\end{array}$ & Only for 2D B-scans images. \\
\hline Chang Tang et al. [24] & $\begin{array}{l}\text { Based on low rank matrix } \\
\text { representation. }\end{array}$ & $\begin{array}{l}\text { Use the similarity between } \\
\text { different patches. }\end{array}$ & $\begin{array}{l}\text { Faster than dictionary } \\
\text { Based techniques }\end{array}$ & High computational cost \\
\hline $\begin{array}{l}\text { Saba Adabi et al. } \\
\qquad[25]\end{array}$ & $\begin{array}{l}\text { Learnable despeckling } \\
\text { framework }\end{array}$ & $\begin{array}{l}\text { This technique use the auto } \\
\text { encoder neural network } \\
\text { with filter selection process }\end{array}$ & $\begin{array}{l}\text { a. Use only figure of merit as } \\
\text { a quality metric. } \\
\text { b. Improve the filter } \\
\text { selection process. }\end{array}$ & $\begin{array}{l}\text { Only five quality parameter } \\
\text { decide the result }\end{array}$ \\
\hline
\end{tabular}

\section{Conclusion}

This paper highlighted study about the different despeckling methods used for quality enhancement of retinal optical coherence tomography (OCT) images. The above study shows that among the different type of despeckling methods are used for OCT images. Study shows that an approach based on bilateral filter removes the significant amount of speckle noise while preserves the edges of the images. The Wavelet transform based approach is also a better solution for speckle noise reduction because of its multiscale nature. Out of different ICA techniques, SOBI is the one of the best techniques used for speckle noise reduction. Further work can be carried out in the variational image decomposition approach, because this approach is separate the speckle noise from the cartoon part and the texture part of the OCT images. Finally, learnable despeckling framework is also helpful for the selection of digital filter as per the requirement of the images features.

\section{References}

1. Huang D, Swanson EA, Lin CP, Schuman JS, Stinson WG, et al. (1991) Optical coherence tomography. Science 254(5035): 1178-1181.

2. Hee MR, Puliafito CA, Wong C, Duker JS, Reichel E, et al. (1995) Quantitative assessment of macular edema with optical coherence tomography. Archives of ophthalmology 113(8): 1019-1029.

3. Koprowski R, Wróbel Z (2011) Image processing in optical coherence tomography using Matlab. Poland: Katowice.

4. Podoleanu AG (2005) Optical coherence tomography. The British Journal of Radiology 78(935): 976-988.

5. Drexler W, Fujimoto JG (2008) Optical Coherence Tomography, Springer, USA.
6. Chen Y, Vuong LN, Liu J, Ho J, Srinivasan VJ, et al. (2009) Threedimensional ultra high resolution optical coherence tomography imaging of age-related macular degeneration. Opt Express 17(5): 40464060 .

7. Krebs I, Hagen S, Brannath W, Haas P, Womastek I, et al. (2010) Repeatability and reproducibility of retinal thickness measurements by optical coherence tomography in age-related macular degeneration. Ophthalmology 117(8): 1577-1584.

8. Schmitt, Joseph M, Xiang SH, Kin MY (1999) Speckle in optical coherence tomography. Journal of biomedical optics 4(1): 95-105.

9. Lee JS (1980) Digital image enhancement and noise filtering by use of local statistics. IEEE Transactions on Pattern Analysis and Machine Intelligence 2(2): 165-168.

10. Kuan DT (1985) Adaptive noise smoothing filter for images with signaldependent noise. IEEE Transactions on Pattern Analysis and Machine Intelligence 2(1): 165-177.

11. Frost VS, Stiles JA, Shanmugan KS, Holtzman JC (1992) A model for radar images and its application to adaptive digital filtering of multiplicative noise. IEEE Transactions on Pattern Analysis and Machine Intelligence 4(2): 157-166.

12. Leyuan F, Shutao Li, Qing Nie, Joseph AI, Cynthia A, et al. (2012) Sparsity based denoising of spectral domain optical coherence tomography images. Biomedical Optics Express 3(5): 927-942.

13. Leyuan F, Shutao Li, Ryan PM, Qing N, Anthony NK, et al. (2013) Fast Acquisition and Reconstruction of Optical Coherence Tomography Images via Sparse Representation. IEEE Transactions On Medical Imaging 32(11): 2034-2049.

14. Anantrasirichaia N, Nicholsonb L, James EM, Irina E, Katie M, et al. (2014) Adaptive-weighted bilateral filtering and other pre-processing techniques for optical coherence tomography. Computerized Medical Imaging and Graphics 38(6): 526-539. 
15. Yongzhao Du, Gangjun Liu, Guoying Feng, Zhongping Chena (2014) Speckle reduction in optical coherence tomographyimages based on wave atoms. Journal of Biomedical Optics 19(5): 056009.

16. Jinming D, Wenqi Lu, Christopher T, Irene G, Frank P, et al. (2016) Denoising optical coherence tomography using second order total generalized variation decomposition. Biomedical Signal Processing and Control 24: 120-127.

17. Sudeep PV, Issac SN, Palanisamy P, Jeny R, Xiaojun Y, et al. (2016) Enhancement and bias removal of optical coherence tomography images: An iterative approach with adaptive bilateral filtering. Computers in Biology and Medicine 71: 97-107.

18. Hongwei R, Lei Q, Xinjun Zhu (2016) Speckle reduction and cartoontexture decomposition of ophthalmic optical coherence tomography images by variational image decomposition. Optik 127(19): 7809-7821.

19. Ahmadreza B, Roshan MD, Zeyun Yu (2016) Application of Independen Component Analysis techniques in speckle noise reduction of retinal OCT images. Optik 127(15): 5783-5791.

20. Raheleh K, Hossein R, Ivan S (2015) Three Dimensional Data-Driven Multi Scale Atomic Representation of Optical Coherence Tomography. IEEE Transactions on Medical Imaging 34(5): 1042-1062.
21. Zahra A, Hossein R (2016) Statistical Modelling of Retinal Optical Coherent Tomography. IEEE Transactions on Medical Imaging 35(6): $1544-1554$

22. Leyuan F, Shutao Li, David C, Sina Farsiu (2017) Segmentation Based Sparse Reconstruction of Optical Coherence Tomography Images. IEEE Transactions on Medical Imaging 36(2): 407-421.

23. Muxingzi Li, Ramzi I, Biswarup C, Wolfgang H (2017) Statistical model for OCT image denoising. Biomed Opt Express 8(9): 3903-3917.

24. Chang T, Lijuan C, Jiajia C, Xiao Z (2017) Speckle noise reduction for optical coherence tomography images via non-local weighted group low-rank representation. Laser Physcis Letter 14(5).

25. Saba A, Elaheh R, Anne C, Hamed MK, Silvia C, et al. (2018) Learnable despeckling framework for optical coherence tomography images. Journal of Biomedical Optics 23(1): 1-12.

\section{(a) (i) \\ This work is licensed under Creative Commons Attribution 4.0 License}

To Submit Your Article Click Here: Submit Article

DOI: 10.32474/OAJBEB.2018.01.000109

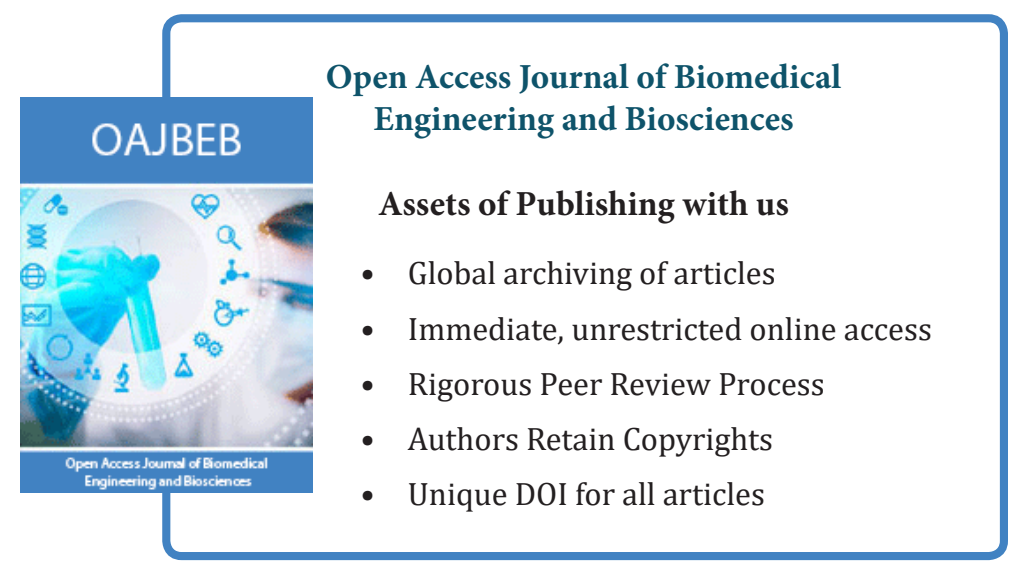

